\title{
Introducing Extended DEMO Construction Model to RPA Application
}

\author{
Xiaohan Tian ${ }^{1+}$, Junichi Iijima ${ }^{2}$ \\ ${ }^{1,2}$ Tokyo Institute of Technology
}

\begin{abstract}
Business Process technology has long been discussed in various business process methodologies with diverse perspectives. Among them, the discussions on were mainly limited only in effectively fulfilling functional, informational and organizational aspects. For enriching the discussion scope, from DEMO (Design and Engineering Method for Organizations) perspective, this research extends to capture both human interactions and human capabilities aspects in the business process and the relating modeling methodology is also provided. For contextualizing our proposal, RPA (Robotic Process Automation), as one of the typical new application in freeing human workers from tedious and repetitive tasks, is adopted as an illustration example of our artifact development. Also, our proposed modeling methodology is evaluated in a deep-dive field study with a Chinese Bank X to testify the utility in analyzing and identifying applicable tasks for RPA adoptions and eventually delivering the business process model.
\end{abstract}

Keywords: RPA (Robotic Process Automation), DEMO (Design and Engineering Method for Organizations), Business Process Management, Business Process Automation

\section{Introduction}

As a management discipline, Business Process Management concentrates on exploring how to leverage business processes to achieve organizational goals by reinforcing the ongoing process performance and the harnessing of the essential business process [1]. Furthermore, a set of structured methodologies in multiple perspectives on business process management have been developed. For instance, Process Analysis provides a range of tools and techniques in prioritizing process details, measuring process performances and then empowering decision making [2]. Business Process Modelling (i.e., Business Process Modelling Notation, UML activity diagram) focuses on integrating views from informational, functional and organizational aspects of the business activities in organizations [3].

Moreover, with the advancement of information and technology, diverse business process technology has been developed to achieve productivity enhancement in organizations. One of the most renowned fields is Business Process Automation. For automating conceivable parts of the business process, Business Process Automation tools are widely adopted in the automation of simple process such as facilitating coordination between different tasks [4]. In order to effectively automate a business process, it is required to create an relevant Business Process Model in the beginning, aiming at presenting certain aspects of the business process for further automation development. However, among these modelled aspects in facilitating Business Process Automation, few of them showed applicability in grasping and depicting the claimed contributions of the business process technology in improving work efficiency. Therefore, without appropriate Business Process Models created for automation, organizations struggle in understanding the necessities in initiating the automation their AS-IS business processes.

In order to deal with this type of struggle in Business Process Automation, figuring out a suitable Business Process Modelling technique is the starting point.

\footnotetext{
+ Corresponding author. Tel.: +817038205678

E-mail address: tian.x.aa@m.titech.ac.jp
} 
Specifically, this technique should first specialize in conceptualizing productivity enhancements achieved by business process technology and followed by depicting the conceptualization in the Business Process Model. Therefore, the first research question that we proposed is, 1) how to conceptualize the productivity enhancement by business process technology?

DEMO (Design and Engineering Method for Organizations) is a Business Process Modelling methodology, well-known for its ability of conceptualizing ,1) the human interactions aspect; 2) the human capabilities aspect, of a business process and providing a set of Business Process Models. From these two aspects, the original discussions in DEMO provided differentiation features between the process that can be facilitated by business process technology and the process that can only be manually operated [5]. Therefore, we fixated the research focus on DEMO, and endeavoured to investigate its capabilities on delivering a Business Process Model which illustrates the productivity contribution by business process technology.

In the investigation of DEMO, we first comprehended its discussions on the differentiation of business processes that can be facilitated by technology and the ones that can only be handled by human workers. However, the discussions in DEMO have not been transformed into corresponding methodology for Business Process Modelling. And hence, it left us the second research gap and proposed the second research question, 2) from DEMO perspective, how to model the productivity enhancement by business process technology?

In the development of modelling methodology, we stemmed from the original Business Process Modelling techniques provided by DEMO. By adding the concerns of business process technology from DEMO theoretical part, the Extended DEMO Construction Model was developed. For illustrating and evaluating the developed artefact, by having deep-dive field study with a Chinese Bank X, we created an illustration case of the latest application in Business Process Automation, RPA (Robotic Process Automation). The relating discussion on the utility of our modelling methodology is also presented.

\section{Literature Review}

We started this research by reviewing the present researches on Business Process Modelling methodologies in facilitating the application of business process technologies. After confirming DEMO as the most appropriate methodology in conceptualizing and depicting productivity enhancement of business process technology, we proceeded to review a technology application, RPA (Robotic Process Automation), to demonstrate its applicability in illustrating and evaluating our proposal.

\subsection{Business process modelling methodology}

In Business Process Management, managerial efforts are usually paid on the effective business process models to provide a comprehensive understanding of the ongoing business activities and enable the following analysis [6]. According to Aguilar-Saven [6]), before modelling business processes, the modelling purpose of the business process depends on the abstraction requirements of the target organization. For instance, the purpose of modelling a business process can be understanding and analysing business process [7]. Specifically, for descriptive aim, Flow Chart technique is for establishing a logical sequence of a set of business activities [8]. Also, concentrating on the responsibility specification and human interaction in the business processes, RAD (Role activity diagrams) [9] and RID (Role interaction diagrams) [10] provide more details of the business process. Also, the Gantt Chart introduced a new dimension, time scale, to manage the work duration performance of business process models [11].

On the other hand, inspired by traditional software development methodologies, numerous techniques have been applied in business process technologies [12], for instance, Data Flow techniques especially present the data flowing in different subjects of the business activities[13], while UML (Unified Modelling Language) is utilized in modelling both static and dynamic areas of software systems[14] . Further, for enabling multiple purposes of structuring, analysis, and simulation at the same time, the modelling techniques have been developed further, among them, Workflow technique is renowned for its modelling in information transmission among different stakeholders by following predefined rules in business process technologies [15]. On the other hand, Coloured- Petri-Net (CPN) is a technique that orients the transitions of actions and states of the business process and enables the communications between different systems in 
business process technologies [16]. Also, in both academic research and practical use of business process technologies, BPMN (Business Process Modelling Notation) is renowned for its flexible embeddedness of any new process elements and the improved understanding shared by IT and businesspeople [17].

However, if concerning with the productivity enhancement brought by business process technologies, none of the above modelling techniques provide structured conceptualization and relating models. As a result, we found DEMO (Design and Engineering Method for Organizations), a Business Process Modelling methodology that provides conceptualization on how business process technologies can contribute to business processes in organizations. From the perspectives of human interactions and human capabilities, DEMO provides theoretical discussions in differentiating business processes that can be facilitated by technologies and the ones that can only be implemented by human. Therefore, this differentiation enables the conceptualization of productivity enhancement by business process technologies.

Meanwhile, we also found that, the discussions of business process technologies in DEMO have not been transformed into relating methodologies of creating business process models. Therefore, after achieving full conceptualization of productivity enhancement by DEMO theoretical discussions, we started to develop the corresponding Business Process Modelling techniques stemming from the original DEMO methodologies. For better illustrating and evaluating our developed methodology, we decided to discuss the modelling procedures of it in the context of the latest application of Business Process Automation, RPA (Robotic Process Automation).

\subsection{Robotic process automation (RPA)}

For attaining a comprehensive understanding of RPA, we first reviewed a set of definitions from both academic types of research and practitioner proposals. Irrelevant to physical robots, Robotic Process Automation, widely known as RPA, is now playing as a virtual worker in the form of a software solution and aims to take over the previous business tasks handled by human workers. As was initially defined by the industry practitioners, RPA was introduced to the public mainly through the functional requirements that different solution vendors can manage to realize. Among them, in terms of the characteristics of RPA, some argue that activities that RPA involve in are mainly in 3 aspects, 1) automating business processes; 2) working across different IT- system; 3) manipulate data [18]. When working across different IT system, what RPA can do is imitating the previous human actions and it means no interferences are caused to the existing operations due to the introduction of RPA [18]. The others stress on that the automation and the following maintenance procedures in RPA do not require the executors to have any programming skills since it can be completed only in interface level [19]. For highlighting the contributions that RPA can bring about to the organization, RPA is further differentiated from Business Process Management Systems (BPMSs) and Intelligent Automation. Unlike Business Process Reengineering in which business processes are expected to be re-conceptualized and re-engineered and multiple functional packages like database are embedded, as a light deployment required software solution as RPA, it majors in automating the existing business processes while no database section is attached in RPA[19]. Also, in comparison to intelligent automation, RPA software excels at automating rule-based tasks without the capabilities to handle exceptions that do not scheme in the predefined rules.

The above review helped us to confirm the applicability of RPA to serve as the illustration example of our developed modelling methodology for two reasons, 1) as the latest application, RPA is calling for researches and discussions; 2) its claim on freeing and optimizing human capabilities in organizational works match the theoretical statement of human capabilities in DEMO.

\section{Theoretical background: DEMO (Design and Engineering Method for Organizations)}

This section presented the theoretical background of DEMO approach in human interactions and human capabilities aspects. Moreover, the original modeling methodology was also introduced.

\subsection{Human interactions}


In DEMO, organizational work is completed by human interactions. Moreover, these human interactions are categorized by their purpose as,1) Production is delivering products or services to the environment as requested, and a Production fact is created by executing a relating Production act;2) Coordination is making the efforts on communication and negotiation for delivering products, and a Coordination fact is created by executing the Coordination act [20]. Therefore, for all Coordination that serves for one Production purpose, the combination of this coordination and production are defined as a Transaction [20]. For executing each Transaction, the primary responsibility unit is defined as an Actor role. For Actor role who initiates the transaction, he or she is called as an Initiator and Executor for who execute the transaction. With these constructive elements, DEMO then provides a set of procedures of creating a Construction Model which illustrates the highest abstraction level of a business process model in the construction view.

In the application of business process technologies, from DEMO perspective, it majors in mimicking the Production Acts of human beings in handling repetitive business tasks in large amount while proceeding to take over more works in Coordination Acts.

\subsection{Human capabilities}

On the other hand, DEMO also investigates the human capabilities performed in a business process. For the capability that can create original new things, it is classified as Performa capability, and this capability is entitled only to human beings, which cannot be imitated by any technologies[20]. Then, the capability of coping with the content of existed information is defined as Informa[20]. Moreover, the capability of dealing with the format of the existed information is defined as Forma[20]. Therefore, in a business process, Performa capability is considered as the most superior capability of human in organizational environments and is supported by Informa and Forma; and Informa is defined as the second superior capability for its second higher complexity level in operations and is supported by Forma with the lowest complexity level.

Correspondingly, the Transactions that deliver products by using Performa capability are defined as Original Transaction; the Transactions that deliver products by using Informa capability are defined as Informational Transaction; the Transactions that deliver products by using Forma capability are defined as Documental Transaction.

In the application of business process technologies, from the perspective of DEMO, they are not replacing but enhancing the Informa and Forma capabilities of human workers in automating repetitive and tedious works, so that human workers' capabilities can be optimized and shifted to Performa works.

\subsection{Original construction model}

In line with the human interactions and human capabilities perspectives, the original Construction Model was created to illustrate the most concise analysis result of a business process. In this original Construction Model, Actor roles of both Initiators and Executors ( $A_{\mathrm{i}}$ in Figure 1) are identified and differentiated by Initiator link and Executor link (as illustrated in Figure 1). Also, it introduces a Composite actor role to represent the subjects from outside of the organization. However, Transaction in Construction Model only presents the Production aspects but omits the Coordination aspects of a business process. Further, by using the boundary of the organization to separate the areas of interest in a business process, the original Construction Model only concerns about activities in which Performa capabilities are performed.

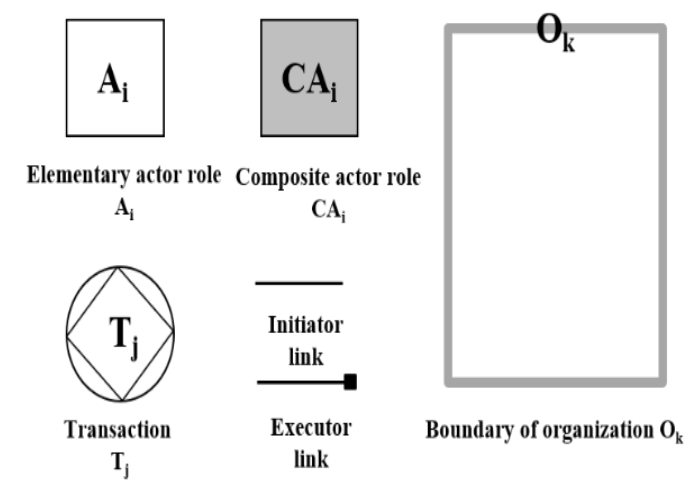

Figure.1: Legend of Construction Model [20] 


\section{Artifact Development: Extended DEMO Construction Model}

As what is presented in section 3, business process technologies major in reinforcing Informa and Forma capabilities of human workers performed in a business process. Therefore, based on the original Construction model and theoretical perspectives in human interactions and human capabilities, in this section, we developed an Extended Construction Model to elucidate the Informa and Forma aspects of a business process and their relations with Performa aspect.

\subsection{The modeling methodology of Extended DEMO construction model}

The original Construction Model does not present the Coordination aspect of a business process, for maintaining this modeling feature, we also omitted the discussion on Coordination aspect of a business process in the developed model.

For highlighting the business process technology contributions of enhancing human capabilities discussed in section 3.2. In addition to the original Performa capability, both the Informa and Forma capability enhanced in Production aspect of a business process are illustrated. The extension was achieved by step 4 and step 6 in Figure 2. In step 4, based on the Production Facts generated by each Transaction, the Transactions can be classified into, 1) Original Transaction; 2) Informational Transaction; 3) Documental Transaction. Specifically, recalling the explanation in section 3.2, Performa is identified as the most superior capability entitled to human and followed by Informa and Forma. In one Transaction, among all created Production Facts, we focused on the performed capability of the highest superiority level and classified the Transaction based on it.

Moreover, in step 6, for highlighting the differentiations between different capabilities performed, the connection links between different types of Transactions are also identified to illustrate the relationships between Actor roles and Transactions.

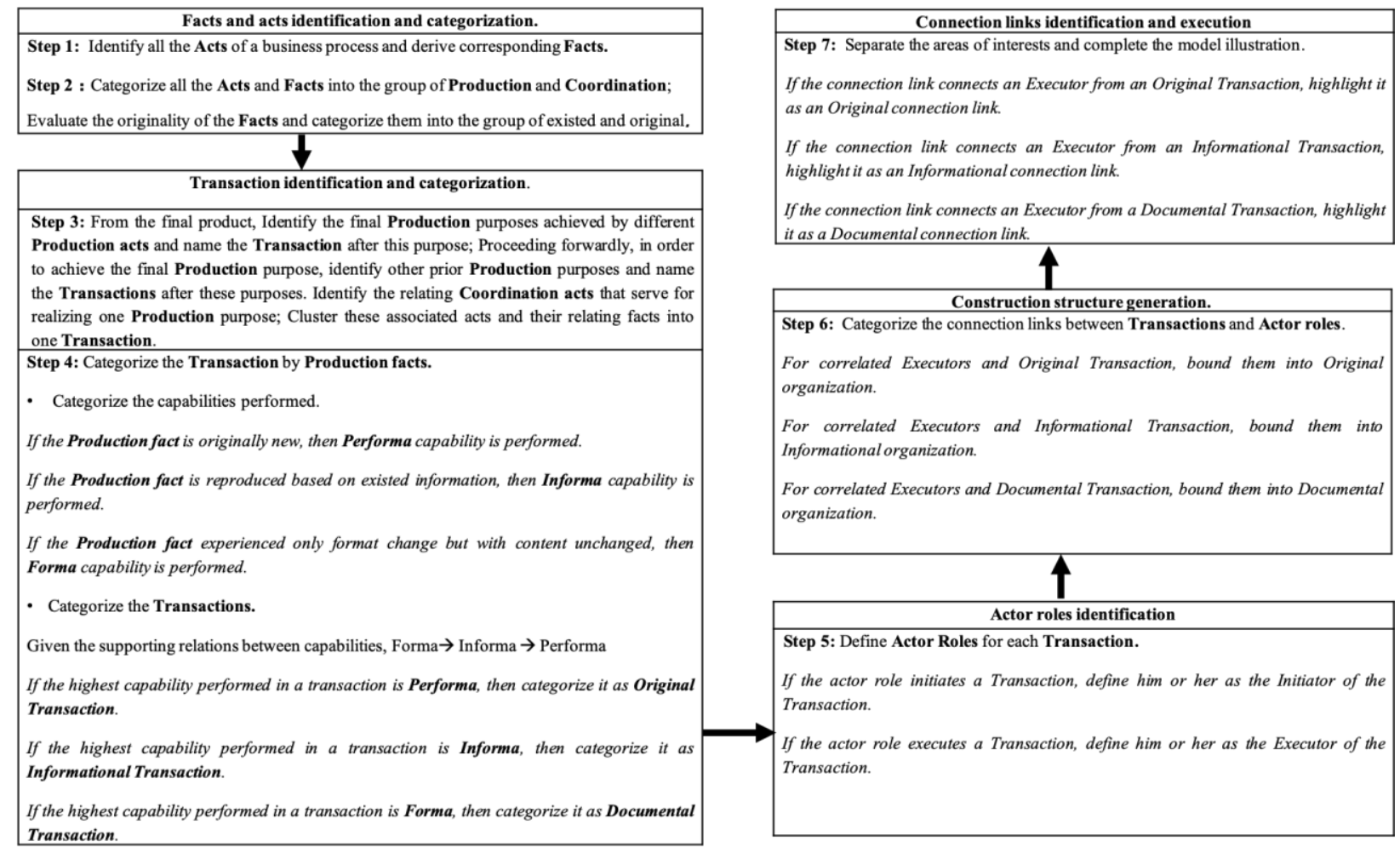

Figure.2: The modeling methodology of Extended DEMO Construction Model

\subsection{Naming principles of the extended construction model of DEMO}

In construction perspective, DEMO defines actor roles as the primary responsibility unit in a Transaction and then provides two types of actor roles, Initiator and Executor related to the Transaction. For the actor role who is responsible for executing the transaction, namely, the executor, he or she is named after the transaction to highlight this responsibility. For instance, executor of "membership registration" is named as "membership register." Meanwhile, the numbering consistency between executor and transaction is also 
maintained. For instance, "A1" refers to "Actor role 1" and is responsible for executing "T1", namely, Transaction 1.

\section{Artifact Illustration and Evaluation in a Field Study of a Chinese Bank $X$}

In this section, we evaluated the Extended DEMO Construction Model by conducting a deep-dive field study with a Chinese Bank X. We first presented the field study context as follows,

Bank X was planning to introduce RPA to its financial department. Although their RPA solution provider has already proposed business process automation plan to their process of publishing invoice report as a pilot project, the financial department management was not completely convinced by the plan for two reasons, 1) since they had fully qualified employees to deliver satisfying outcomes, why they had to apply RPA to the AS-IS process? 2) RPA solution providers only appealed their available RPA techniques, but would these techniques genuinely make contributions to the AS-IS process?

In this context, we created Extended DEMO Construction Model for their process of publishing invoice report in Bank $\mathrm{X}$, aiming at providing a systematic business process model in highlighting potential contributions that can be brought by RPA in the human capabilities and human interaction aspects of the process.

\subsection{Case illustration: publishing an invoice report}

The AS-IS process of publishing an invoice report started by a back-office agent of the financial department. Also, the case description is as follows,

The back-office agent first selects invoice documents to be verified and move all of them into the same folder. For all these documents, he then sorts out the required data and inputs them into an Excel file. After that, he logs into the invoice verification website and inputs the collected data from the Excel file. And, he verifies the data on this platform and inputs the verified data into another Excel file. Based on the checking and calculating the verified data, the back-office agent generates the invoice report and closes the process.

This AS-IS process was illustrated by a flowchart in Figure 3 .

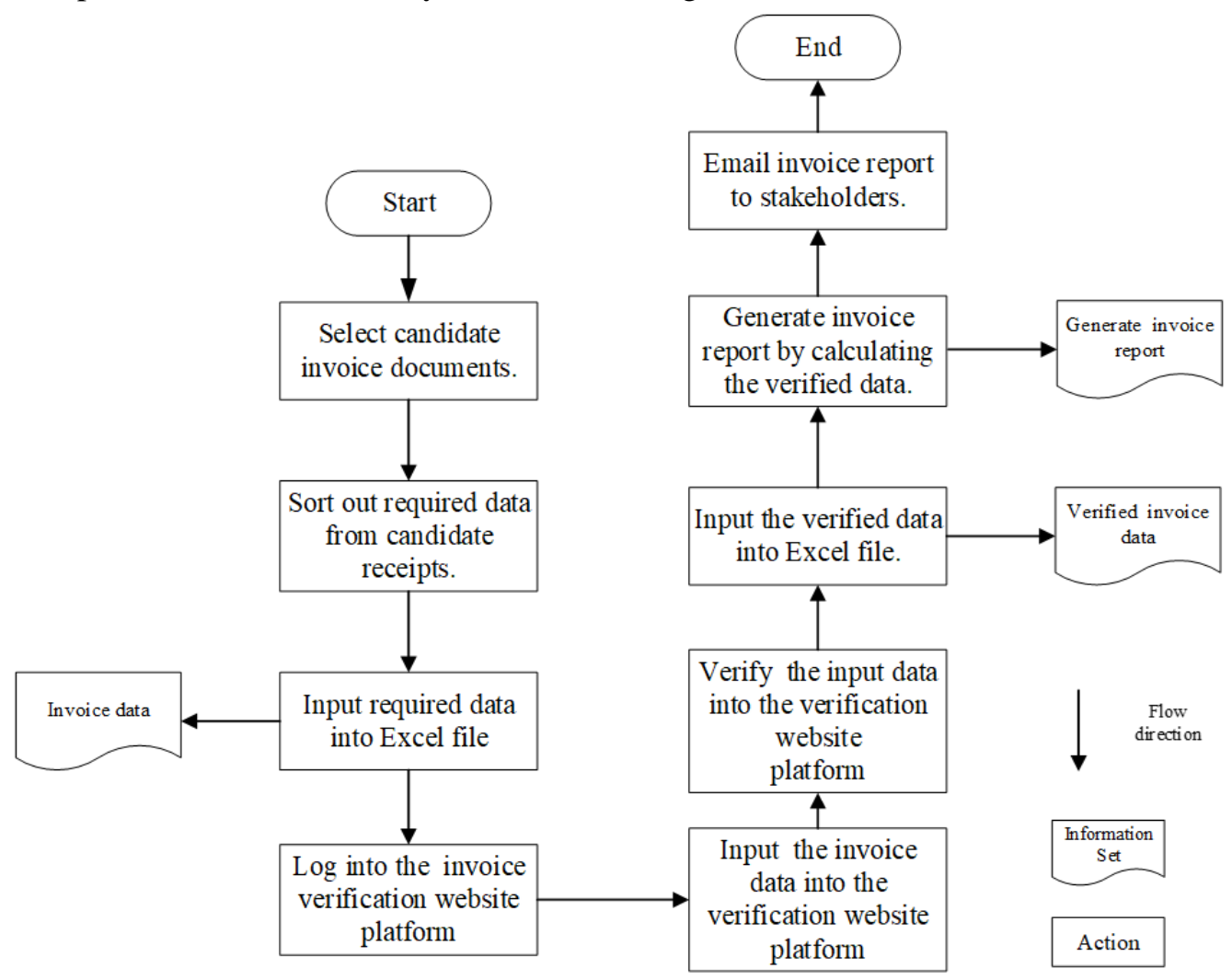

Figure.3: Flowchart of publishing invoice report 


\section{Step 1: Identify all the Acts of a business process and derive corresponding Facts.}

In the first step, we picked out every action shown as steps in Figure 3 and identified them as Acts in DEMO approach. Then, a corresponding Fact was derived by executing an Act, and we highlighted this accomplishment of action by describing the facts in Present Perfect Tense as shown in Table 1. Differing from the Flowchart methodology, in DEMO approach, we indicated the actor of each Act from the beginning, for instance, in the case of publishing invoice report, the back-office agent is the only actor of all Acts.

Step 2: Categorize all the Acts and Facts into the group of Production and Coordination; Evaluate the originality of the Facts and categorize them into the group of existed and original.

In Step 2, we first categorized all the Acts and Facts that we identified and derived in step 1 into either Production or Coordination. If the Act was completed only by one actor, for instance, Back-office agent selects candidate invoice documents, this type of Act and the Fact produced by executing this Act was categorized as Production. On the other hand, if the Act is required to involve more than one actor, we considered Coordination activities happened between them to deliver products collaboratively. In the process of publishing an invoice report, only the last Act made by the back-office agent involved the email receivers as the other actor. We then identified this Act as making a statement in Coordination activities.

When evaluating the originalities of Facts, we added more discussions with Bank X to situate our developed artifacts into their cases. Also, according to the discussion, the selection of candidate invoice documents did not have standardized rules to refer. Instead, the selection was handled under specific situations in their practical business scenarios.

Table.1: Fact (F) and Act(A) of publishing invoice report

\begin{tabular}{|l|l|}
\hline \multicolumn{1}{|c|}{ Acts } & \multicolumn{1}{|c|}{ Facts } \\
\hline Back-office agent selects candidate invoice documents. & Candidate invoice documents have been selected. \\
\hline Back-office agent sorts out required data. & Required data has been sorted out. \\
\hline Back-office agent inputs required data to an Excel file. & Required data has been input to an Excel file. \\
\hline Back-office agent logs into the verification platform. & Invoice verification platform has been logged into. \\
\hline $\begin{array}{l}\text { Back-office agent inputs the required data to the invoice } \\
\text { verification platform. }\end{array}$ & Required data has been input to the platform. \\
\hline Back-office agent verifies the input data on the platform. & Input data has been verified. \\
\hline Back-office agent inputs the verified data into an Excel file. & Verified data has been input into an Excel file. \\
\hline Back-office agent generates the invoice report. & Invoice report has been generated. \\
\hline Back-office agent emails the invoice report to stakeholders. & Invoice report has been emailed. \\
\hline
\end{tabular}

Also, therefore, in the process of publishing an invoice report, Bank $X$ stressed the originalities of selected invoice documents could only brought only by human workers. Therefore, we categorized the Fact, Candidate invoice documents have been selected, as an original Fact. This original fact was highlighted in the shadow area in Table 2. Also, based on these documents, the rest facts could be created by either manipulating existed data or dealing with the format of the data, which did not contribute to originality creation. We then classified the rest of the Facts as existed Facts. The analysis result for step 2 is shown in Table 2.

Step 3: From the final product, Identify the final production purposes achieved by different Production acts and name the Transaction after this purpose; Proceeding forwardly, in order to achieve the final Production purpose, identify other prior Production purposes and name the Transactions after these purposes. Identify the relating Coordination acts that serve for realizing one Production purpose; Cluster these associated acts and their relating facts into one Transaction.

We started with identifying Production purpose of this process. In the beginning, since the final product as the result of the process is presented, it is affirmative to identify the last Production purpose initially. 
The last Production purpose was identified as publishing the invoice report. So therefore, the third transaction was named as T3 Invoice publication. For completing this publication, it was required to first accomplish the Production on publishing the report by calculating verified data. However, for Coordination acts, we did find out that, the completion of the invoice report publication was sending the generated reports to relating stakeholders. And this Coordination act was therefore attributed to Transaction 3.

Table.2: Act and Fact types in publishing an invoice report

\begin{tabular}{|c|c|c|}
\hline Acts/Facts types & Acts & Facts \\
\hline \multirow{8}{*}{ Production } & $\begin{array}{l}\text { Back-office agent selects candidate } \\
\text { invoice documents. }\end{array}$ & $\begin{array}{l}\text { Candidate invoice documents have been } \\
\text { selected. }\end{array}$ \\
\hline & $\begin{array}{l}\text { Back-office agent sorts out } \\
\text { required data. }\end{array}$ & Required data has been sorted out. \\
\hline & $\begin{array}{l}\text { Back-office agent inputs required } \\
\text { data to an Excel file. }\end{array}$ & $\begin{array}{l}\text { Required data has been input to an } \\
\text { Excel file. }\end{array}$ \\
\hline & $\begin{array}{l}\text { Back-office agent logs into the } \\
\text { invoice verification platform. }\end{array}$ & $\begin{array}{l}\text { Invoice verification platform has been } \\
\text { logged into. }\end{array}$ \\
\hline & $\begin{array}{l}\text { Back-office agent inputs the } \\
\text { required data to the platform. }\end{array}$ & $\begin{array}{l}\text { Required data has been input to the } \\
\text { platform. }\end{array}$ \\
\hline & $\begin{array}{l}\text { Back-office agent verifies the input } \\
\text { data on the platform. }\end{array}$ & Input data has been verified. \\
\hline & $\begin{array}{l}\text { Back-office agent inputs the } \\
\text { verified data into an Excel file. }\end{array}$ & $\begin{array}{l}\text { Verified data has been input into an } \\
\text { Excel file. }\end{array}$ \\
\hline & $\begin{array}{l}\text { Back-office agent creates the } \\
\text { invoice report. }\end{array}$ & Invoice report has been created. \\
\hline Coordination & $\begin{array}{l}\text { Back-office agent emails the } \\
\text { invoice report to stakeholders. }\end{array}$ & Invoice report has been emailed. \\
\hline
\end{tabular}

For publishing the invoice report, the first prior production purpose is verifying the data to be published. And therefore, we named the second transaction as T2 Invoice verifying. Since the verification was executed on the online platform, the Production act started from logging into this verification platform. Then, the data verification was completed by inputting the required data and had them verified in the platform. And the verified results were stored in an Excel file. Regarding the Coordination acts that serve for achieving this production purpose, the original process description did not show the necessities of coordinating with other actors, and we maintained this feature for Transaction 2.

Before the data can be verified, due to the data verification scope, it is required to select data from the raw datasets in documents. Therefore, the second prior Production purpose is selecting invoice data. And we then named the first Transaction after this purpose, T1 Invoice selecting. In this Transaction, to prepare these invoice data, it is required to start from the Production act of selecting candidate invoice documents followed by sorting out required data out of these documents. Eventually, the data to be verified were stored in an Excel file. For the same reason with Transaction 2, the Coordination acts were omitted to be discussed. The result of step 3 analysis was shown in Table 3.

Table. 3: Transactions of publishing a payment receipt

\begin{tabular}{|c|c|c|}
\hline Production acts & Coordination acts & Transactions \\
\hline $\begin{array}{l}\text { Back-office agent selects candidate invoice } \\
\text { documents. }\end{array}$ & \multirow[b]{3}{*}{ Omitted in the process } & \multirow[t]{3}{*}{ T1 Invoice selecting } \\
\hline Back-office agent sorts out required data. & & \\
\hline $\begin{array}{l}\text { Back-office agent inputs required data to an Excel } \\
\text { file. }\end{array}$ & & \\
\hline $\begin{array}{l}\text { Back-office agent logs into the invoice verification } \\
\text { platform. }\end{array}$ & \multirow{4}{*}{ Omitted in the process } & \multirow[t]{4}{*}{ T2 Invoice verifying } \\
\hline $\begin{array}{l}\text { Back-office agent inputs the required data to the } \\
\text { platform. }\end{array}$ & & \\
\hline $\begin{array}{l}\text { Back-office agent verifies the input data on the } \\
\text { platform. }\end{array}$ & & \\
\hline $\begin{array}{l}\text { Back-office agent inputs the verified data into an } \\
\text { Excel file. }\end{array}$ & & \\
\hline Back-office agent creates the invoice report. & $\begin{array}{l}\text { Back-office agent emails the } \\
\text { invoice report to } \\
\text { stakeholders. }\end{array}$ & T3 Invoice report publishing \\
\hline
\end{tabular}




\section{Step 4: Categorize the Transaction by Production fact.}

As explained in section 3.2, in a business process, human capabilities of Performa, Informa, and Forma support each other to complete business tasks. The originality brought by Performa activities is supported by Informa activities in dealing with information content. Meanwhile, the manipulation of information content is supported by handling information format by Forma activities. For highlighting this supporting relation, in this step, the Transactions are classified based on the highest capability performed in Production Acts of a Transaction.

Recalling the original fact identified in step 2, selected candidate invoice documents, represented that all Coordination and Production occurring in Transaction 1 were to deliver this original Fact. And therefore, Transaction 1, Invoice selecting, was classified as an Original Transaction.

Further work in this step was to categorize Transaction 2 and Transaction 3. For Transaction 2, the Production Facts created was, verified invoice data, which was obtained based on the calculation of the existed invoice data and no original Production Facts were created. And thus, in the second transaction, the highest capability performed is Informa, and is then identified as Informational Transaction.

As for Transaction 3, we found out that the report was generated by filling out the verified invoice data into the report templates and was then sent to stakeholders. So, it was affirmative that no content change occurred, and no originality was brought in Transaction 3, but only the verified data was transferred from Excel file to a PDF file and then to email. So, therefore, in Transaction 3, Forma is the highest capability performed and is then identified as Documental Transaction. The analysis result of step 4 was shown in Table 4.

Table.4: Transaction types of publishing a payment receipt

\begin{tabular}{|l|l|}
\hline \multicolumn{1}{|c|}{ Transactions } & Transaction types \\
\hline T1 Invoice selecting & Original \\
\hline T2 Invoice verifying & Informational \\
\hline T3 Invoice publishing & Documental \\
\hline
\end{tabular}

Step 5: Define Actor Roles for each Transaction.

To identify the responsibility unit in each transaction, in this step, we first named the executor for each transaction after the responsibility performed to deliver the products. From T1 Invoice selecting, the executor was named as Invoice selector. And for T2 Invoice verifying and T3 Invoice publishing, the executors were named as Invoice selector and Invoice publisher, respectively.

Based on identified executors, we proceeded to identify initiators. For the first transaction, we found it was self-activated and thus identified the initiator also as Invoice selector. After the first transaction was accomplished, the Invoice selector continued to initiate the second transaction of Invoice verifying. Eventually, the last transaction was initiated by Invoice verifier when the verification was accomplished. Table 5 showed the analysis result of step 5 .

Table.5: Actor roles of publishing a payment receipt

\begin{tabular}{|l|l|l|}
\hline \multicolumn{1}{|c|}{ Transactions } & \multicolumn{1}{c|}{ Initiators } & \multicolumn{1}{c|}{ Executors } \\
\hline T1 Invoice selecting & Invoice selector & Invoice selector \\
\hline T2 Invoice verifying & Invoice selector & Invoice verifier \\
\hline T3 Invoice publishing & Invoice verifier & Invoice publisher \\
\hline
\end{tabular}

Step 6: Categorize the connection links between Transactions and Actor roles.

The categorization of connection links in this step lies in the types of transactions that the Executors and Initiators involve. By this categorization rule, we identified the connection link as Original link for T1 Invoice selecting between Initiator as Invoice selector and Executor as Invoice selector. While connection link for T2 Invoice verifying between Invoice selector and Invoice verifier was identified as Information link, Documental link was identified for T3 Invoice publishing. Table 6 showed the analysis result for this step. 
Table.6: Connection links of publishing payment receipt

\begin{tabular}{|l|l|l|l|}
\hline \multicolumn{1}{|c|}{ Initiators } & \multicolumn{1}{c|}{ Transactions } & \multicolumn{1}{c|}{ Executors } & Connection links types \\
\hline Invoice selector & T1 Invoice selecting & Invoice selector & Original \\
\hline Invoice selector & T2 Invoice verifying & Invoice verifier & Informational \\
\hline Invoice verifier & T3 Invoice publishing & Invoice publisher & Documental \\
\hline
\end{tabular}

\section{Step 7: Separate the areas of interests and complete the model illustration.}

The last step is to summarize the analysis result and complete the model illustration. In this step, we separated the areas of interests by three types of Organizations as explained in section 3.2,1) Original organization; 2) Informational organization;3) Documental organization. These organizations were constituted by different transactions and relating actor roles and linked by connection links.

For Transaction 1 as an original transaction, together with Invoice selector as both of its initiator and executor constituted the Original organization. And Informational organization contained Transaction 2 and its executor while Transaction 3 and its executors were in Documental organization. The final model illustration was illustrated in Figure 4, and the Extended DEMO Construction Model legends were also shown.

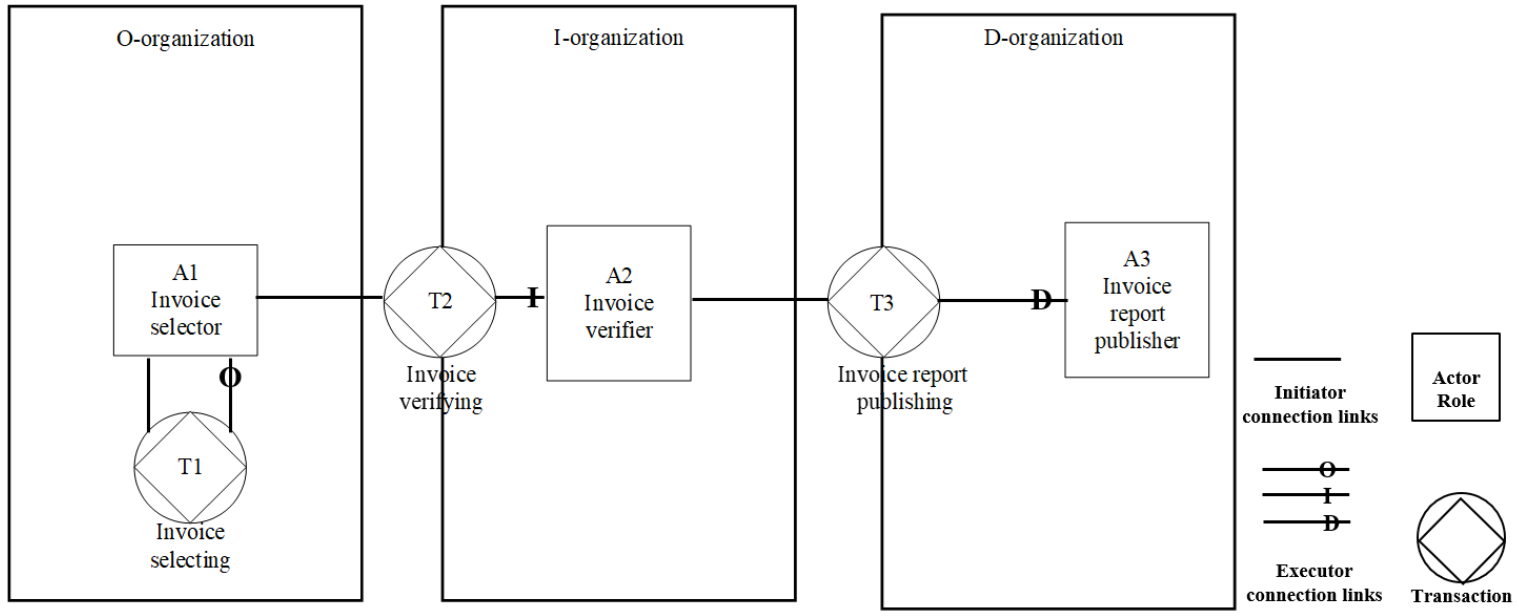

Figure.4: The Extended DEMO Construction Model of publishing an invoice report

\subsection{Evaluation and discussion on the field study}

Based on the steps presented in section 4, we delivered the analysis results to Bank X and attempted to deal with their current two concerns on the adoption of RPA.

1) Why their current smooth manual operations of publishing an invoice report had to be taken over by RPA?

From the perspective of human interactions, in this process, by analyzing step 2, we confirmed that most of the acts required in the operations were productions accomplished only by one Actor Role. Only the last step of sending emails to stakeholders had to include more than one Actor Role. Therefore, all these operations demonstrated light dependencies on the other Actor Roles, which created the capacity for business process technologies like RPA to complete the tasks alone and deliver timely task results in large amounts.

On the other hand, if taking the aspect of human capabilities, except for Transaction 1, both Transaction 2 and 3 showed less necessities for human involvements to ensure originality. Also, this low requirements on human worker contributions also left spaces for business process technologies like RPA to deliver repetitive task results of higher accuracy rates.

Therefore, the answer to this concern was that compared with the previously developed manual operations, the adoption of RPA was likely to bring about productivity enhancement by upscaling task amounts and improved accuracy rates on tasks of low originalities created by human workers.

2) How to evaluate if the automation plan provided by RPA service provider can enhance the current performance? 
Based on Figure 4, we suggested that Bank X consider automating only Transaction 2 and Transaction 3. This suggestion was different from the automation plan from their RPA service provider. According to their plan, Transaction 1 was also suggested to be automated since they only recognized Transaction 1 as a Documental Transaction in which invoice documents were selected based on specific rules and have no impact to the data content. Nevertheless, by having deep-dive discussions with Bank X, we identified the originality creation requirements in Transaction 1 did exist. Since RPA cannot provide proper ad-hoc invoice selections to specific scenarios, we suggested Bank X keep this transaction operated by human workers.

\section{Conclusion and Limitations}

In this paper, from the perspective of DEMO, we accomplished the conceptualization of productivity enhancement brought by business process technology. Also, we extended and enriched the discussions of the Business Process Modelling methodologies in the application of business process technologies, in the aspects of human interactions and human capabilities. As a result, the filed study with Chinese Bank X enabled us to illustrate our proposal in a practical context and the following evaluation demonstrated the utility of our developed methodology.

To conclude this research, we provided answers to the two research questions in this section.

\section{1) How to conceptualize the productivity enhancement by business process technology?}

There are two aspects that business process technologies potentialize in improving organizational work, 1) human interaction; 2) human capabilities. By enhancing human interactions in coordination and production, RPA may enhance productivity by upscaling task amounts. Meanwhile, by enhancing human capabilities in Informa and Performa, RPA may improve the accuracy rate of tasks.

2) From DEMO perspective, how to model the productivity enhancement by business process technology?

A viable methodology is the Extended DEMO Construction Model developed in this research. Especially for potential users, creating this model can enable them to compare their essential needs with the automation plan provided by business process technology service providers and eventually understand their genuine needs on business process automation.

The limitations of this research mainly came from the lack of rigorous academic research materials in RPA field. Due to this shortage, the research proposal of this research might be insufficient in arguments demonstrations. Meanwhile, the evaluation of developed artifacts is expected to be executed with more practical cases to testify its utility in various business process technology contexts.

\section{Reference}

[1] J. Jeston and J. Nelis, Business process management. Routledge, 2014.

[2] Á. Rebuge and D. R. Ferreira, "Business process analysis in healthcare environments: A methodology based on process mining," Information systems, vol. 37, no. 2, pp. 99-116, 2012.

[3] B. Scholz-Reiter and E. Stickel, Business process modeling. Springer Science \& Business Media, 2012.

[4] M. Dumas, M. La Rosa, J. Mendling, and H. A. Reijers, Fundamentals of business process management. Springer, 2013.

[5] J. L. Dietz, Enterprise ontology: theory and methodology. Springer Science \& Business Media, 2006.

[6] R. S. Aguilar-Saven, "Business process modelling: Review and framework," International Journal of production economics, vol. 90, no. 2, pp. 129-149, 2004.

[7] K. T. Phalp, "The CAP framework for business process modelling1," Information and Software Technology, vol. 40, no. 13, pp. 731-744, 1998.

[8] R. Lakin, N. Capon, and N. Botten, "BPR enabling software for the financial services industry," Management services, vol. 40, no. 3, pp. 18-20, 1996.

[9] A. W. Holt, H. Ramsey, and J. Grimes, "Coordination system technology as the basis for a programming environment," Electrical Communication, vol. 57, no. 4, pp. 307-314, 1983. 
[10] G. Booch, Object oriented analysis \& design with application. Pearson Education India, 2006.

[11] R. Aguilar-Saven, "Business process modelling techniques and tools," Department of Production Economics. WP291, Linköping Sweden, 2001.

[12] K. Phalp and M. Shepperd, "Quantitative analysis of static models of processes," Journal of Systems and Software, vol. 52, no. 2-3, pp. 105-112, 2000.

[13] Q. Li and Y.-L. Chen, "Data flow diagram," in Modeling and Analysis of Enterprise and Information Systems: Springer, 2009, pp. 85-97.

[14] N. Russell, W. M. van der Aalst, A. H. Ter Hofstede, and P. Wohed, "On the suitability of UML 2.0 activity diagrams for business process modelling," in Proceedings of the 3rd Asia-Pacific conference on Conceptual modelling-Volume 53, 2006, pp. 95-104: Australian Computer Society, Inc.

[15] L. Fischer, New Tools for New Times-the Workflow Paradigm: The Impact of Information Technology on Business Process Reengineering. Future Strategies, Incorporated, 1994.

[16] K. Jensen, Coloured Petri nets: basic concepts, analysis methods and practical use. Springer Science \& Business Media, 2013.

[17] S. A. White, BPMN modeling and reference guide: understanding and using BPMN. Future Strategies Inc., 2008.

[18] P. Lowes, "An Introduction to Robotic Process Automation," Available: http://deloitte.wsj.com/cio/2016/03/13/anintroduction-to-robotic-process-automation/

[19] M. Fulton, S. O’Sullivan, and S. Rayment, "Robotic Process Automation - friend or foe for your risk profile?," Australia2016.

[20] J. L. Dietz, What is Enterprise Ontology? Springer, 2006. 